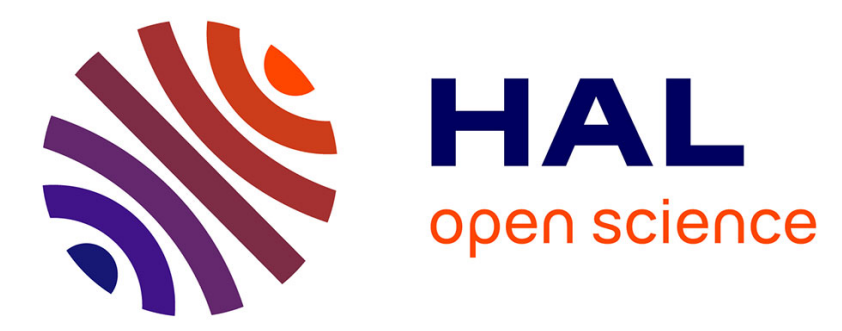

\title{
Thermo-electro-hydrodynamic instabilities in a dielectric liquid under microgravity
}

Satish Malik, Harunori Yoshikawa, Olivier Crumeyrolle, Innocent Mutabazi

\section{To cite this version:}

Satish Malik, Harunori Yoshikawa, Olivier Crumeyrolle, Innocent Mutabazi. Thermo-electrohydrodynamic instabilities in a dielectric liquid under microgravity. Acta Astronautica, 2012, 81, pp.563-569. 10.1016/j.actaastro.2012.06.023 . hal-00906503

\section{HAL Id: hal-00906503 https://hal.science/hal-00906503}

Submitted on 21 Nov 2013

HAL is a multi-disciplinary open access archive for the deposit and dissemination of scientific research documents, whether they are published or not. The documents may come from teaching and research institutions in France or abroad, or from public or private research centers.
L'archive ouverte pluridisciplinaire HAL, est destinée au dépôt et à la diffusion de documents scientifiques de niveau recherche, publiés ou non, émanant des établissements d'enseignement et de recherche français ou étrangers, des laboratoires publics ou privés. 


\title{
Thermo-electro-hydrodynamic instabilities in a dielectric liquid under microgravity
}

\author{
Satish V. Malik, Harunori N. Yoshikawa, Olivier Crumeyrolle \\ \& Innocent Mutabazi \\ Laboratoire Ondes et Milieux Complexes, UMR 6294, CNRS-Université du Havre \\ 53, rue de Prony, BP540 - 76058 Le Havre Cedex, France
}

\begin{abstract}
Linear stability analysis of a dielectric fluid confined in a cylindrical annulus of infinite length is performed under microgravity conditions. A radial temperature gradient and a high alternating electric field imposed over the gap induce an effective gravity that can lead to a thermal convection even in the absence of the terrestrial gravity. The linearized governing equations are discretized using a spectral collocation method on Chebyshev polynomials to compute marginal stability curves and the critical parameters of instability. The critical parameters are independent of the Prandtl number, but they depend on the curvature of the system. The critical modes are non-axisymmetric and made of stationary helices.
\end{abstract}

Keywords: Electrohydrodynamics, Heat transfer, Convection

\section{Introduction}

A combined action of a radial temperature gradient and an electric field on a dielectric liquid is of great interest from both fundamental research and applications. The temperature gradient induces a gradient of the permittivity $\epsilon=\epsilon(T)$ and the latter is coupled with the electric field to produce an electric body force [1]. When the electric field is alternating with a high frequency, i.e., with a time-period much shorter than the charge relaxation time, there is no free charge in the liquid [2] and the density of the electric force is given by

$$
\mathbf{f}_{e}=-\frac{1}{2} E^{2} \boldsymbol{\nabla} \epsilon-\nabla\left[\frac{1}{2} \rho E^{2}\left(\frac{\partial \epsilon}{\partial \rho}\right)_{T}\right],
$$

where $E$ is the local electric field and $\rho$ is the liquid density. The first and second terms are called the dielectrophoretic and electrostrictive forces, respectively. As the first term is related to the temperature gradient, it can develop thermal convection through a thermoelectro-hydrodynamic instability without any other external force, e.g., the terrestrial gravity. From the fundamental standpoint, this convection considered in a cylindrical or spherical geometry is of particular interest. With applying a radial temperature gradient and electric field, the dielectrophoretic force is aligned in the radial direction and can be deemed as an effective gravity that we call the electric gravity. Many geophysical problems can be simulated by this convection in the dielectric liquid $[3,4]$. The application of the temperature gradient and the electric field may be used for heat transfer enhancement in dielectric liquids and may yield large reductions in weight and volume of heat transfer systems. This technique is attractive for aerospace cooling systems [5].

In the present paper, we consider the configuration of an annulus filled by a dielectric liquid and subjected to a radial temperature gradient and a radial alternating electric field. The stability in this configuration was investigated by Chandra \& Smylie [6] with assuming the microgravity environment where the buoyancy associated with the terrestrial gravity was absent. They considered the linear stability to axisymmetric disturbances and found instability above a critical electric Rayleigh number $R a_{c}$. Chandra \& Smylie took into account only disturbances in the velocity and temperature fields in their analysis. No disturbance in the electric field was considered, although the permittivity (and electric field) would have perturbation components through its temperature dependence. This possible feedback effect of the temperature disturbances on the electric permittivity was taken into account by Takashima [7] by including the 
Gauss's law for the electric field in the linear stability problem. He considered the problem in the same annular geometry for axisymmetric disturbances. His analysis showed the critical Rayleigh number depends on another parameter $\beta$ representing the thermal variation of the permittivity. Besides these theoretical stability analyses in the annular geometry, some works exist for the spherical geometry [8, 9, 10]. Yavorskaya et al. [8] considered a dielectric liquid layer between two concentric spherical surfaces with a radial temperature gradient and a radial electric field. They found instability occurrence and a sensitive dependence of the critical Rayleigh number on the radius ratio of the two surfaces. Futterer et al. [10] performed a numerical simulation by a spectral code for cases with and without the rotation of the inner surface. They found development of steady convection as well as periodic and chaotic behavior of the flow. When the rotation is absent, they found sudden transition from steady to chaotic flows.

In the literature, some experiments are found that were performed in the annular geometry. Chandra \& Smylie [6] reported an experiment conducted under the terrestrial gravity condition. They used vertically installed concentric cylinders with the gap filled by a silicone oil. When $R a$ exceeded a critical value $R a_{c}$, they observed the rapid increase of the Nusselt number from the unity. The experimental $R a_{c}$ agreed well with their theoretical result under the microgravity condition. However, there will be a thermal convection due to the terrestrial gravity in the basic state $[11,12]$. Further justification is needed to conclude that this Nusselt number behavior is associated to the TEHD instability. Recently, Sitte et al. [13] conducted an experiment in microgravity conditions. They visualized the thermal field in the gap of two cylinders by the Schlieren technique and observed convection at large electric Rayleigh numbers. Their results indicate the non-axisymmetric behavior of flow, which has never been considered in the stability analysis for the annular geometry.

The present work deals with linear stability to nonaxisymmetric as well as axisymmetric disturbances. In Section 2, we present the governing dynamical and electric equations. The results are given in Section 3. The last section is concerned with the discussion and conclusion.

\section{Governing Equations}

We consider an incompressible Newtonian dielectric fluid confined between two coaxial cylinders of radii $R_{1}$ and $R_{2}\left(R_{1}<R_{2}\right)$, maintained at temperatures $T_{1}$ and $T_{2}$ $\left(T_{1}>T_{2}\right)$, respectively. A high frequency alternating electric tension is imposed over the gap. As the liquid does not have enough time to react to the rapid field variations, only the effective field from the mean value $\boldsymbol{E}(\boldsymbol{r})$ pertains to the electric force (1).

We adopt a Boussinesq-type approximation: thermal variation of fluid properties influences the dynamics only through the destabilizing electric body force (1). As to the permittivity, we assume a linear dependence on the temperature:

$$
\epsilon(T)=\epsilon_{2}\left[1-e\left(T-T_{2}\right)\right]
$$

where $\epsilon_{2}$ is the electric permittivity at temperature $T_{2}$ and $e$ is the thermal coefficient of permittivity. The equations governing the flow subjected to a temperature gradient and an alternating electric field are [1],

$$
\begin{gathered}
\boldsymbol{\nabla} \cdot \boldsymbol{v}=0, \\
\frac{\partial \boldsymbol{v}}{\partial t}+(\boldsymbol{v} \cdot \nabla) \boldsymbol{v}=-\frac{1}{\rho} \boldsymbol{\nabla} P+v \Delta \boldsymbol{v}+\frac{1}{2} \frac{\epsilon_{2} e}{\rho} E^{2} \boldsymbol{\nabla} T, \\
\frac{\partial T}{\partial t}+(\boldsymbol{v} \cdot \boldsymbol{\nabla}) T=\kappa \Delta T, \\
\boldsymbol{\nabla} \cdot(\epsilon \boldsymbol{E})=0, \\
\boldsymbol{E}=-\boldsymbol{\nabla} \phi,
\end{gathered}
$$

where $v$ is the kinematic viscosity, $\kappa$ is the thermal diffusivity, $v$ is the velocity. The generalized pressure $P$ includes the electrostriction component:

$$
P=p+\frac{1}{2} \rho\left(\frac{\partial \epsilon}{\partial \rho}\right)_{T} E^{2} .
$$

We take the gap size $d=R_{2}-R_{1}$ as characteristic length and $\tau_{0}=d^{2} / v$ as characteristic time as in Takashima [7]; it represents the shortest timescale for most of dielectric liquids. The characteristic velocity is given by $V=v / d$, correspondingly. The temperature is nondimensionalized by temperature difference $\Delta T=T_{1}-T_{2}$ between the cylindrical walls. Further the pressure is scaled by $P_{0}=\rho v^{2} / d^{2}$ and the electric field by $E_{0}=V_{e} / d$, where $V_{e}$ is the effective value of the potential difference applied across the cylinders. The scaling leads to a set of four dimensionless parameters necessary to specify the flow: the dimensionless curvature $\delta=d / R_{1}$, the Prandtl number $\operatorname{Pr}=v / \kappa$, the thermal variation of permittivity over the imposed temperature difference $\beta=e\left(T_{1}-T_{2}\right)$ and the electric Grashoff number:

$$
G r=\frac{g_{e} \alpha \Delta T d^{3}}{v^{2}} \text { with } g_{e}=\frac{\epsilon_{2} e V_{e}^{2}}{2 \rho \alpha d^{3}},
$$


where $g_{e}$ is the electric gravity. The parameter $\beta$ has been omitted in $[6,8]$ by assuming either a small coefficient $e$ or a small temperature difference. We will also use later the electric Rayleigh number $R a=G r P r$.

When the imposed temperature and electric potential differences are small, the electric gravity is colinear with the temperature gradient, so that there is no source of vorticity and velocity fields. The basic state will then be purely conductive without fluid motion: $\boldsymbol{v}=0$. For an infinite cylindrical annulus, the basic state is expected to be axisymmetric and axially invariant, i.e., $\boldsymbol{E}=-(d \Phi / d r) \mathbf{e}_{r}$ and $T=T(r)$. Thus it is governed by the following nondimensionalized equations obtained from Eqs. (3b)-(3e)

$$
\begin{gathered}
\frac{d P}{d r}-G r\left(\frac{d \Phi}{d r}\right)^{2} \frac{d T}{d r}=0, \\
\frac{1}{r} \frac{d}{d r}\left(r \frac{d T}{d r}\right)=0, \\
\frac{1}{r} \frac{d}{d r}\left(r \epsilon \frac{d \Phi}{d r}\right)=0,
\end{gathered}
$$

with the boundary conditions

$$
\begin{array}{ll}
T\left(r_{1}\right)=1 \quad \text { and } & T\left(r_{2}\right)=0, \\
\Phi\left(r_{1}\right)=1 & \text { and } \quad \Phi\left(r_{2}\right)=0,
\end{array}
$$

where $r_{1}$ and $r_{2}$ are the dimensionless radii of the inner and outer cylinders, respectively: $r_{1}=\delta^{-1}, r_{2}=1+\delta^{-1}$. The integration of these equations yields the temperature and the electric potential of the basic state as

$$
T(r)=-\frac{\log \left(\frac{\delta}{1+\delta} r\right)}{\log (1+\delta)} \quad \text { and } \quad \Phi(r)=\frac{\log [1+\beta T(r)]}{\log (1-\beta)} .
$$

The generalized pressure of the basic flow is obtained by substituting these solutions into equation (6a):

$$
P=G r \int\left(\frac{d \Phi}{d r}\right)^{2} \frac{d T}{d r} d r .
$$

The stability of the flow can be examined by considering linearized governing equations about the basic state with respect to infinitesimal perturbation expanded into the normal mode:

$\boldsymbol{\Psi}(r) \exp [i k z-i n \varphi+s t] \quad$ with $\quad \boldsymbol{\Psi}=[u, v, w, \pi, \theta, \phi]^{T}$,

where $k$ is the axial wavenumber and $n$ is the azimuthal mode number. In general, the time evolution rate of the perturbation $s$ is complex : $s=\sigma+i \omega$ where $\sigma$ and $\omega$ are the temporal growth rate and frequency respectively. The perturbative velocity components in the radial, azimuthal and axial directions are $u, v$ and $w$ respectively. The perturbations of the generalized pressure, the temperature and the electric potential are denoted by $\pi, \theta$ and $\phi$. Substituting the normal mode into the governing equations, subtracting the basic flow and neglecting the nonlinear terms in perturbation, we obtain the following linearized equations:

$$
\begin{gathered}
\frac{1}{r} \frac{d}{d r}(r u)-\frac{i n}{r} v+i k w=0, \\
\Delta u-\frac{u}{r^{2}}+\frac{2 i n}{r^{2}} v-\frac{d \pi}{d r}+G r\left[\left(\frac{d \Phi}{d r}\right)^{2} \frac{d \theta}{d r}+2 \frac{d \Phi}{d r} \frac{d T}{d r} \frac{d \phi}{d r}\right]=s u, \\
\Delta v-\frac{v}{r^{2}}-\frac{2 i n}{r^{2}} u+\frac{i n}{r} \pi-\frac{i n}{r} G r\left(\frac{d \Phi}{d r}\right)^{2} \theta=s v, \quad(10 \mathrm{~b}) \\
\Delta w-i k \pi+i k G r\left(\frac{d \Phi}{d r}\right)^{2} \theta=s w, \\
\frac{1}{P r} \Delta \theta-\frac{d T}{d r} u=s \theta, \\
(1-\beta T) \Delta \phi-\beta \frac{d T}{d r} \frac{d \phi}{d r}-\beta\left[\frac{d \Phi}{d r}\left(\frac{d}{d r}+\frac{1}{r}\right)+\frac{d^{2} \Phi}{d r^{2}}\right] \theta=0,
\end{gathered}
$$

with the Laplacian

$$
\Delta=\frac{d^{2}}{d r^{2}}+\frac{1}{r} \frac{d}{d r}-\left(\frac{n^{2}}{r^{2}}+k^{2}\right) .
$$

In the equations of motion (10b)-(10d), the terms including the Grashof number $G r$ stem from the dielectric force term in Eq. (3b). The coupling of electric and thermal fields affects liquid motion. Such coupling is also seen in the terms proportional to $\beta$ in Eq. (10f). The temperature dependence of the permittivity can modify the system behavior through these terms, as Takashima examined its influence on the stability [7]. These couplings contain the radial temperature gradient $d T / d r$, the basic electric field $(-d \Phi / d r)$ and the gradient of the electric field, $\left(-d^{2} \Phi / d r^{2}\right)$. Since these three gradients depend on the curvature of the annulus, the stability of the system will be sensitive to the curvature.

As to boundary conditions, perturbation velocity, temperature and electric potential must vanish on cylindrical surfaces:

$$
u=\frac{d u}{d r}=v=w=\theta=\phi=0 \quad \text { at } r=r_{1}, r_{2} .
$$




\section{Results}

The governing equations (10a)-(10f) is discretized using collocation method $[14,15]$ and considered only at finite number of points $r_{j}$ inside the gap $(j=$ $1,2, \cdots, N-1) . N$ is the highest order of considered Chebyshev polynomials. These points are related to the Chebyshev-Gauss-Lobatto collocation points $x_{j}=$ $\cos (j \pi / N)$ by $r_{j}=\left(x_{j}+1\right) / 2+\delta^{-1}$. The discretized governing equations and the boundary conditions results in a generalized eigenvalue problem:

$$
\mathcal{L} \Psi=s \mathcal{M} \Psi
$$

where the operators $\mathcal{L}$ and $\mathcal{M}$ contains the characteristics on the basic state.

The eigenvalue problem (13) is solved in order to obtain the eigenvalue relation $\mathcal{F}(P r, G r, \eta, n, k, \sigma, \omega)=$ 0 for the parameter ranges $\operatorname{Pr} \in[1,200]$ and $\eta \in$ $[0.1,0.9]$. Marginal stability curves $G r=G r(k)$ is computed for different values of $n$. The critical mode $\left(k_{c}, n_{c}, \omega_{c}\right)$ is then determined from the curves. Figure 1 shows few marginal stability curves for different values of the curvature $\delta$ and a fixed value of the Prandtl number $P r=7$. The lowest minimum belongs to the marginal stability curve with $n=n_{c} \neq 0$, i.e., the critical mode is non-axisymmetric. Moreover, it is observed that the critical modes are stationary $\left(\omega_{c}=0\right)$. Values of the critical parameters from Fig. 1 are given in Table 1 . The values of $k_{c}$ and $n_{c}$ imply the convection rolls of the critical mode are made of helices which are inclined to the azimuthal direction by an angle $\psi=\tan ^{-1}[2 n \delta / k(2+\delta)]$. For $\delta=0.111, \psi=57.3^{\circ}$.

The variation of the critical Grashof number with Prandtl number is elucidated in Fig. 2 for $\delta=0.25$. It is found that the critical values of $G r$ vary inversely with $\operatorname{Pr}$ for a given curvature $\delta$. This means that critical Rayleigh number $R a_{c}$ is constant. In the figure, the values of this critical Rayleigh number $R a_{c}$ are also plotted, showing the instability threshold is characterized by a $R a_{c}$ independent of $P r$. The independence from

Table 1: Critical parameters and the marginal curve minima of axisymmetric mode for different curvatures $(\operatorname{Pr}=7, \beta=0.01)$

\begin{tabular}{cc|ccc|cc}
\multicolumn{2}{c|}{ Curvature } & \multicolumn{3}{c|}{ Critical mode } & \multicolumn{2}{c}{ Axisymmetric mode } \\
& $\delta$ & $k_{c}$ & $n_{c}$ & $G r_{c}$ & $k_{\text {min }}$ & $G r_{\text {min }}$ \\
\hline (a) & 0.111 & 1.69 & 25 & 1075 & 3.12 & 1077 \\
(b) & 0.25 & 1.62 & 12 & 526.7 & 3.12 & 530.6 \\
(c) & 1 & 1.53 & 4 & 171.3 & 3.14 & 183.5 \\
(d) & 4 & 1.41 & 2 & 71.37 & 3.25 & 98.31
\end{tabular}
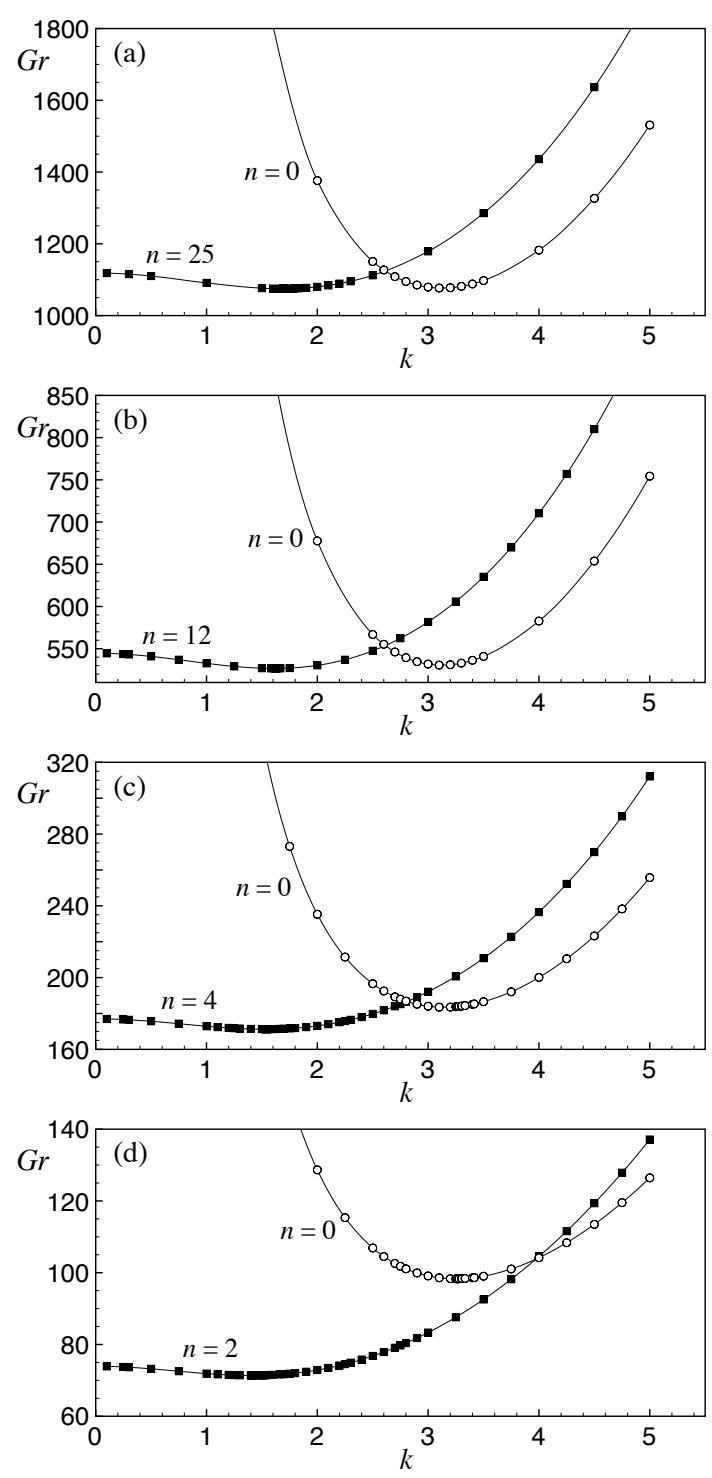

Figure 1: Marginal stability curves for axisymmetric $(n=0)$ and nonaxisymmetric $\left(n=n_{c} \neq 0\right.$ ) perturbations for $\operatorname{Pr}=7, \beta=0.01$ and various values of curvature $\delta$ : (a) $\delta=0.111$, (b) $\delta=0.25$, (c) $\delta=1$ and (d) $\delta=4$.

the Prandtl number is also found in the critical axial wavenumber $k_{c}$ and the critical azimuthal mode number $n_{c}$ (Fig. 3). It is also seen that $k_{c}$ is always around 1.5 and becomes smaller with increasing $\delta$, in contrast with $n_{c}$ which decreases substantially with $\delta$. The influence of the curvature $\delta$ on the critical Rayleigh number $R a_{c}$ is shown in Fig. 4. The value of $R a_{c}$ decreases with $\delta$, i.e., the curvature lowers the instability threshold. This would be related to the fact that the electric field and temperature gradient take locally large values 
at the inner cylindrical surface for large curvatures: the dielectrophoretic body force becomes consequently important.

\section{Discussion}

The obtained results are different from those reported in Chandra \& Smylie [6] and Takashima [7] who considered only axisymmetric disturbances. Therefore, the criterion given by these authors to predict the instability has to be extended to non-axisymmetric perturbations.

The independence of the critical parameters from the Prandtl number is also found in the ordinary RayleighBénard problem, in which a liquid layer between two horizontal plates is heated from below. The critical value of the Rayleigh number $R a^{\prime}=g \alpha \Delta T^{\prime} d^{\prime 3} / \kappa v\left(\Delta T^{\prime}\right.$ : temperature difference, $d^{\prime}$ : depth of the layer, $g$ : gravitational acceleration) is 1708 and the critical wavenumber $k_{c}^{\prime}$ is 3.117, independent of $\operatorname{Pr}$ [16]. The main differences of the TEHD instability problem considered in the present work from this Rayleigh-Bénard problem is that the electric gravity depends on electric force and geometry, while the gravity is constant in the latter problem. As a consequence, the critical parameters in the present problem depend sensitively on the curvature (Figs. 3 $\& 4)$. Such a sensitive dependence is also found in the TEHD instability problem in a spherical geometry [8]. In the ordinary Rayleigh-Bénard problem, the behavior above the criticality depends on $\mathrm{Pr}$, e.g., the occurrence of zigzag, cross-roll and bimodal instabilities found in the nonlinear regime is influenced by $\operatorname{Pr}$ [16]. One may expect such dependence on the Prandtl number in the TEHD instability.

According to our results, under microgravity conditions, it is easier to trigger critical modes for an annulus with a large curvature, i.e., a system of cylindrical surfaces with largely different radii. In Table 2, values of the electric tension $V_{e}$ required to provoke the TEHD instability are shown for different curvatures in two dielectric liquids. The corresponding electric gravity $g_{e}$ is also presented for the gap $d=5 \mathrm{~mm}$. Considered liquids are the Baysilone ${ }^{\circledR}$ silicone oil M5 and the 1-Nonanol, which were used in the GeoFlow experiments [3, 4] and should be used in future experiments with annular geometries in microgravity conditions. The imposed temperature difference $\Delta T$ is assumed to be 5 degrees. The critical parameters $\left(k_{c}, n_{c}, R a_{c}\right)$ for both liquids take similar values for the same curvature $\delta$, as these parameters are independent of $\mathrm{Pr}$. Small differences in values of $R a_{c}$ are due to the difference in the thermal variation of the permittivity $\beta$. It is seen that $V_{e}$ at large curvature is substantially lower than $V_{e}$ at small curvature. By

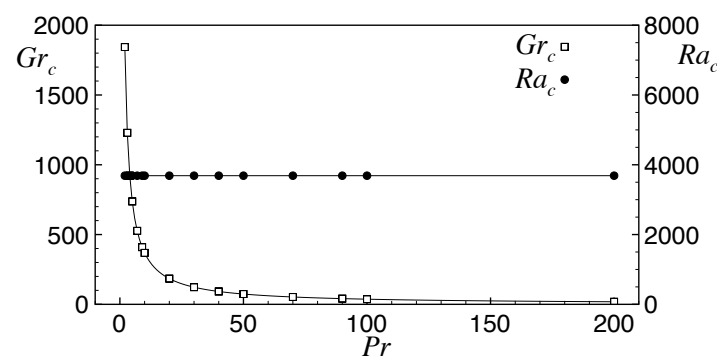

Figure 2: Variation of critical Grashof number $G r_{c}$ and critical Rayleigh number $R a_{c}$ with Prandtl number $\operatorname{Pr}$ for $\delta=0.25$ and $\beta=0.01$.
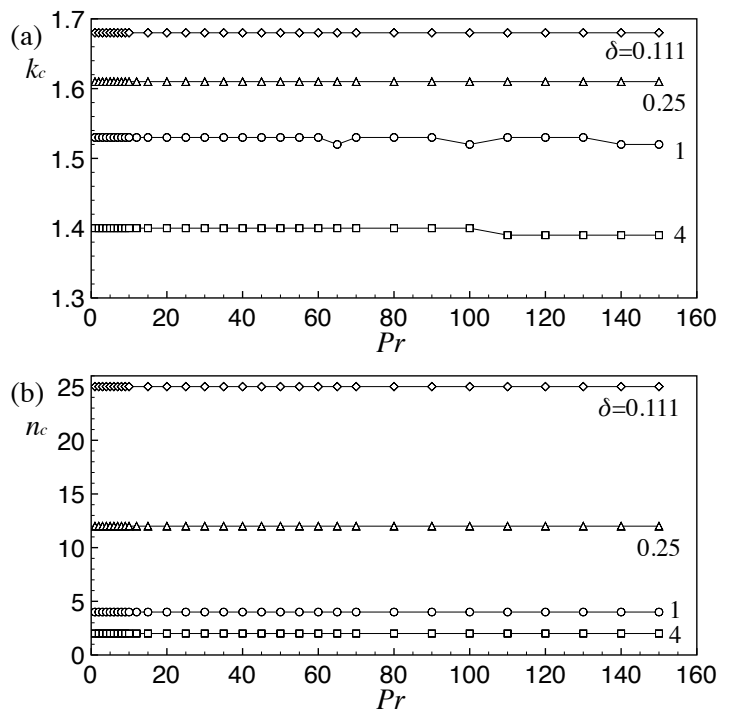

Figure 3: Variation of the critical mode with the Prandtl number $\mathrm{Pr}$ for different values of curvature $\delta$. (a) Critical axial wavenumber $k_{c}$ (b) Critical azimuthal mode number $n_{c}$.

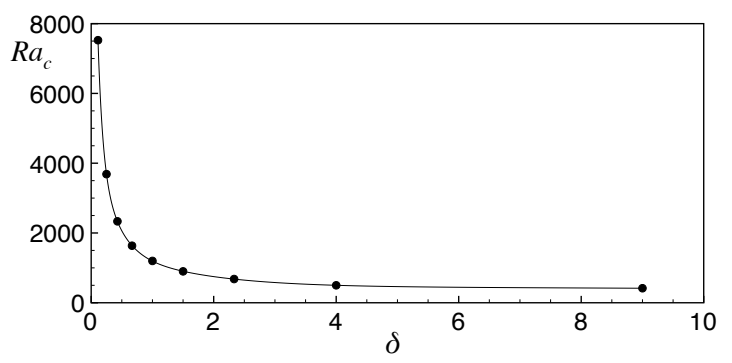

Figure 4: Variation of critical electric Rayleigh number $R a_{c}$ with the curvature $\delta$.

choosing the geometry of $\delta=1$, for example, one can lower $V_{e}$ by a factor 0.4 from the value for $\delta=0.111$. 
Table 2: Few values of the critical electric potential $V_{e}$ for the Baysilone ${ }^{\circledR}$ silicone oil M5 (I) and the 1-Nonanol (II).

\begin{tabular}{c|cccc} 
Liquid & $\delta$ & $R a_{c}$ & $V_{e}(\mathrm{kV})$ & $g_{e}\left(\mathrm{~m} / \mathrm{s}^{2}\right)$ \\
\hline \multirow{3}{*}{ I } & 0.11 & 7785 & 6.60 & 4.46 \\
& 1 & 1206 & 2.60 & 0.691 \\
& 4 & 501 & 1.67 & 0.287 \\
\hline \multirow{3}{*}{ II } & 0.11 & 7254 & 3.38 & 13.8 \\
& 1 & 1192 & 1.37 & 2.27 \\
& 4 & 498 & 0.89 & 0.951
\end{tabular}

I. $\rho=920 \mathrm{~kg} / \mathrm{m}^{3}, v=5 \times 10^{-6} \mathrm{~m}^{2} / \mathrm{s}, \epsilon / \epsilon_{0}=2.7, e=1.065 \times 10^{-3}\left(25^{\circ} \mathrm{C}\right)$

II. $\rho=829 \mathrm{~kg} / \mathrm{m}^{3}, v=14.2 \times 10^{-6} \mathrm{~m}^{2} / \mathrm{s}, \epsilon / \epsilon_{0}=8.83, e=3.03 \times 10^{-2}\left(20^{\circ} \mathrm{C}\right)$

\section{Conclusion}

We have conducted the linear stability analysis of the dielectric fluid confined between two coaxial cylinders and subjected to a radial temperature gradient and a radial alternating electric field. The instability threshold is characterized by the critical Rayleigh number $R a_{c}$. The value of $R a_{c}$ as well as the critical mode $\left(k_{c}, n_{c}\right)$ are independent of the Prandtl number $P r$, while they depend sensitively on the curvature $\delta$. The critical modes are stationary helices $\left(\omega=0, n_{c} \neq 0\right)$. When the curvature $\delta$ is large, the basic state is more unstable to nonaxisymmetric disturbances than to axisymmetric ones.

\section{Acknowledgement}

This work has been partly supported by the CNES (Centre National d'Etudes Spatiales), the CNRS (Centre National de la Recherche Scientifique) and the FEDER (Fonds Européen de Développement Régional).

\section{References}

[1] L. D. Landau, E. M. Lifshitz, Electrodynamics of Continuous Media, 2nd Edition, Vol. 8 of Landau and Lifshitz Course of Theoretical Physics, Butterworth-Heinemann, 1984.

[2] T. B. Jones, Electrohydrodynamically enhanced heat transfer in liquids - A review, Advances in Heat Transfer 14 (1979) 107148.

[3] B. Futterer, M. Gellert, T. von Larcher, C. Egbers, Thermal convection in rotating spherical shell: An experimental and numerical approach within GeoFlow, Acta Astronautica 62 (4-5) (2008) 300-307.

[4] B. Futterer, N. Dahley, S. Koch, N. Scurtu, C. Egbers, From viscous convective experiment 'GeoFlow I' to temperaturedependent viscosity in 'GeoFlow II' - Fluid physics experiments on-board ISS for the capture of convection phenomena in Earth's outer core and mantle, Acta Astronautica 71 (2012) $11-19$.
[5] J. S. Paschkewitz, D. M. Pratt, The influence of fluid properties on electrohydrodynamic heat transfer enhancement in liquids under viscous and electrically dominated flow conditions, Exp. Therm. Fluid Sci. 21 (4) (2000) 187-197.

[6] B. Chandra, D. E. Smylie, A laboratory model of thermal convection under a central force field, Geophysical Fluid Dynamics 3 (1972) 211-224.

[7] M. Takashima, Electrohydrodynamic instability in a dielectric fluid between two coaxial cylinders, Q. J. Mech. Appl. Math. 33 (1) (1980) 93-103.

[8] I. M. Yavorskaya, N. I. Fomina, Y. N. Belyaev, A simulation of central-symmetry convection in microgravity conditions, Acta Astronautica 11 (3-4) (1984) 179-183.

[9] J. E. Hart, G. A. Glatzmaier, J. Toomre, Space-laboratory and numerical simulations of thermal convection in a rotating hemispherical shell with radial gravity, J. Fluid Mech. 173 (1986) 519-544.

[10] B. Futterer, C. Egbers, N. Dahley, S. Koch, L. Jehring, First identification of sub- and supercritical convection patterns from 'GeoFlow', the geophysical flow simulation experiment integrated in Fluid Science Laboratory, Acta Astronautica 66 (2010) 193-200.

[11] M. Ali, P. D. Weidman, On the stability of circular Couette flow with radial heating, J. Fluid Mech. 220 (1990) 53-84.

[12] A. Bahloul, I. Mutabazi, A. Ambari, Codimension 2 points in the flow inside a cylindrical annulus with a radial temperature gradient, Eur. Phys. J. AP 9 (2000) 253-264.

[13] B. Sitte, J. Immohr, O. Heinrichs, R. Maier, C. Egbers, H. Rath, Rayleigh-Bénard convection in dielectrophoretic force fields, in: $12^{\text {th }}$ International Couette Taylor Workshop, Evanston, IL USA, 2001.

[14] S. V. Malik, A. P. Hooper, Three-dimensional disturbances in channel flows, Phys. Fluids 19 (2007) 052102.

[15] C. Canuto, M. Hussaini, A. Quarteroni, T. A. Zang, Spectral Methods in Fluid Dynamics, Springer-Verlang, New York, 1988.

[16] P. G. Drazin, W. H. Reid, Hydrodynamic stability, 2nd Edition, Cambridge University Press, 2004. 